\title{
Ubiquitylation of Drosophila p54/Rpn10/S5a Regulates Its Interaction with the UBA-UBL Polyubiquitin Receptors
}

\author{
Zoltán Lipinszki, Levente Kovács, Péter Deák, and Andor Udvardy* \\ Institute of Biochemistry, Biological Research Centre of the Hungarian Academy of Sciences, H-6701 Szeged, P.O. Box 521, Hungary
}

Supporting Information

ABSTRACT: Analysis of the in vivo ubiquitylation of the p54/Rpn10 polyubiquitin receptor subunit of the Drosophila 26S proteasome revealed that the site of ubiquitylation is the C-terminal cluster of lysines, which is conserved in higher eukaryotes. Extraproteasomal p54 was extensively multiubiquitylated, but only very modest modification was detected in the proteasome-assembled subunit. Ubiquitylation of p54 seriously jeopardizes one of its most important functions, i.e., the interaction of its ubiquitin-interacting motifs with the ubiquitin-like domain of Dsk2 and Rad23 extraproteasomal polyubiquitin receptors. This modification of p54 supports the previous notion that p54 is a shuttling subunit of the $26 \mathrm{~S}$

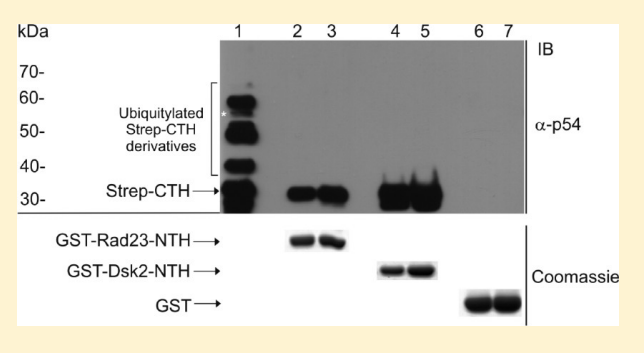
proteasome with a specific extraproteasomal function. This assumption is supported by the observation that, while transgenic p 54 can fully rescue the lethal phenotype of the $\Delta \mathrm{p} 54$ null mutation, its derivative from which the cluster of conserved lysines is deleted shifts the lethality from the early pupa to pharate adult stage but cannot rescue the $\Delta \mathrm{p} 54$ mutation, suggesting that ubiquitylated extraproteasomal p54 has an essential role in the pupa-adult transition.

$\mathrm{T}$ he $26 \mathrm{~S}$ proteasome is responsible for the degradation of the majority of cellular proteins. This large multiprotein complex is assembled in an ATP-dependent reaction from two subcomplexes: the catalytic particle (CP) and the regulatory particle (RP). The catalytic centers of the complex are located in a hidden position, inside an internal chamber of the $\mathrm{CP}$, and this self-compartmentalized arrangement of the site of proteolysis is an important factor controlling the selectivity of the intracellular proteolysis. The narrow diameter of the central channel leading to the catalytic chamber and its gated orifices at the bases of the barrel-shaped CP further contribute to the compartmentalization of the intracellular proteolysis. The RP in cooperation with the ubiquitylating enzyme cascade further extends this selectivity. The ubiquitylating enzyme cascade can recognize specific degradation signals present in proteins ${ }^{1}$ and mark these proteins by the covalent attachment of a polyubiquitin chain. With the participation of polyubiquitin receptors, the RP selectively recognizes and binds the polyubiquitylated proteins, unfolds them via its chaperone-like activity, ${ }^{2,3}$ reprocesses the ubiquitin residues of the substrate proteins, ${ }^{4,5}$ opens the gated channel of the $\mathrm{CP},{ }^{6}$ and feeds them into the CP.

Besides its role in controlled intracellular proteolysis, the ubiquitin-proteasome system is involved in the regulation of a large variety of biological processes, such as the cell cycle, DNA repair, transcription, signal transduction, antigen presentation, garbage disposal, stress response, neurological disorders, etc. ${ }^{7}$ Although the proteolytic activity of the $26 \mathrm{~S}$ proteasome plays a major role in the majority of these processes, components of the $26 \mathrm{~S}$ proteasome may in certain cases exert nonproteolytic activity in the regulatory pathway. Thus, while the proteolytic activity of the $26 \mathrm{~S}$ proteasome is important in the regulation of transcription by the elimination of short-lived transcription factors, its nonproteolytic activities are important for coactivator recruitment, transcriptional elongation, and histone modification. ${ }^{8}$ These observations raise the question of whether individual proteasomal subunits may also be involved in independent, hitherto unknown biological processes. The feasibility of this assumption is based on the observation that a fraction of p54/Rpn10/S5a (Drosophila/yeast/human orthologues), the polyubiquitin receptor subunit of the $26 \mathrm{~S}$ proteasome, exists as a free monomeric protein in Drosophila melanogaster, ${ }^{9,10}$ the yeast Saccharomyces cerevisiae, ${ }^{11}$ and Arabidopsis thaliana. ${ }^{12}$ Subunit p54 has a pivotal role in the selectivity of the intracellular proteolysis as a polyubiquitin receptor, capable of selective recognition and binding of the polyubiquitylated proteins. The extraproteasomal pool of subunit p54 may simply be a purification artifact, a consequence of a weaker association of this subunit with the RP. Our previous findings, however, argue against this simple explanation and suggest an extraproteasomal function of p54. Using a UAS-Gal4-regulated transgenic Drosophila line overexpressing the C-terminal half of subunit p54 (CTH), we have demonstrated the in vivo multiple monoubiquitylation (multiubiquitylation) of the $\mathrm{CTH}$ protein. ${ }^{10}$ In consequence of the lack of the vWA domain present in the $\mathrm{N}$ terminal half $(\mathrm{NTH})$ of subunit p54 and responsible for the assembly of this subunit into the $\mathrm{RP}$, the transgenic $\mathrm{CTH}$ protein was not assembled into the RP. The accumulation of a large quantity of the extraproteasomal $\mathrm{CTH}$, which is accompanied by the appearance of several ubiquitylated derivatives of the $\mathrm{CTH}$, disturbed the developmental regulation of the three major polyubiquitin receptors (Dsk2, Rad23, and p54) of D. melanogaster. ${ }^{10}$

Received: September 7, 2011

Revised: February 27, 2012

Published: February 27, 2012 
This specific postsynthetic modification of the CTH may suggest an extraproteasomal function of subunit p54.

In this work, we identified the site of multiubiquitylation within the $\mathrm{CTH}$, extended the analysis of multiubiquitylation to the fulllength p54 protein, determined the cellular distribution of ubiquitylated and nonubiquitylated full-length p54 proteins, and analyzed the effects of this modification on the interaction of p54 with the extraproteasomal polyubiquitin receptors.

\section{EXPERIMENTAL PROCEDURES}

Sample Preparation. Synchronized ${ }^{10}$ third-instar larvae were collected, washed in PBS, and immediately frozen in liquid nitrogen. The total protein extract was prepared by homogenizing frozen samples for $1 \mathrm{~min}$ at $4{ }^{\circ} \mathrm{C}$ in proteasome buffer $[20 \mathrm{mM}$ Tris-HCl (pH 7.6), $100 \mathrm{mM} \mathrm{NaCl}, 5 \mathrm{mM} \mathrm{MgCl}, 1 \mathrm{mM}$ DTT, $1 \mathrm{mM}$ ATP, and 5\% glycerol] using a micro Potter homogenizer. Debris was removed by centrifugation $\left(16000 \mathrm{~g}\right.$ for $5 \mathrm{~min}$ at $4{ }^{\circ} \mathrm{C}$ ), and supernatants were filtered through sterile fiberglass, immediately mixed with $1 / 3$ volume of $4 \times$ sodium dodecyl sulfate (SDS) sample buffer, and boiled for $4 \mathrm{~min}$ or used directly in different experiments under native conditions. Prior to sizeexclusion chromatography, the protein filtrate was further centrifuged at $100000 \mathrm{~g}$ and $2{ }^{\circ} \mathrm{C}$ for $30 \mathrm{~min}$.

Glutathione $S$-transferase (GST) and GST-tagged Dsk2-NTH or Rad23-NTH were expressed in Escherichia coli DH5 $\alpha$ strains by induction with $0.5 \mathrm{mM}$ IPTG (at an $\mathrm{OD}_{600}$ of $\sim 0.4-0.5$ and $37^{\circ} \mathrm{C}$ for $4 \mathrm{~h})$. Cells were harvested by centrifugation $\left(4{ }^{\circ} \mathrm{C}\right.$ and $2500 \mathrm{~g}$ for $25 \mathrm{~min}$ ), washed in PBS, and disrupted by ultrasonication. Cellular debris was removed by centrifugation $\left(4^{\circ} \mathrm{C}\right.$ and $16000 \mathrm{~g}$ for $5 \mathrm{~min}$ ), and supernatants were frozen in aliquots.

Protein Fractionation and Visualization. Protocols for denaturing SDS-polyacrylamide gel electrophoresis (SDSPAGE) and immunoblotting of the monoclonal [anti-p54/Rpn10 (anti-p54-CTH), anti-p48A/Rpt3, anti-p42A/Rpn7, anti-p39A/ Rpn9, and anti-CP $\alpha 7 / \operatorname{Pros} \alpha 7$ ] and polyclonal (anti-Dsk2, antiRad23, and anti-p54-NTH) antibodies used in our experiments were described previously. ${ }^{10,13}$ The anti-FlagM $\mathrm{M}_{2}$ antibody was from Sigma Aldrich, and anti-ubiquitin, anti-rabbit IgG-HRP, and anti-mouse IgG-HRP antibodies were from Dako (Glostrup, Denmark).

Protein Purification. The larval extracts were prepared in proteasome buffer complemented with complete protease inhibitor cocktail (Roche). In pull-down experiments, Glutathione (GSH) Sepharose (GE Healthcare Life Sciences) beads charged with GST, GST-Dsk2-NTH, or GST-Rad23-NTH recombinant proteins were incubated $\left(4{ }^{\circ} \mathrm{C}\right.$ for $\left.2 \mathrm{~h}\right)$ with protein extracts prepared from 60 Flag-p54-overexpressing thirdinstar larvae (Flag-p54/Dp54-da-Gal4). Unbound proteins were washed out with proteasome buffer followed by elution with $50 \mathrm{mM}$ Tris- $\mathrm{HCl}$ ( $\mathrm{pH} 9.0)$ and $10 \mathrm{mM}$ GSH. Input, flow-through, and eluted (bound) proteins were run on a 9\% SDS-PAGE gel and analyzed by immunoblotting or Coomassie Brilliant Blue staining. Flow-through and bound fractions from GST-Dsk2-NTH and GST-Rad23-NTH pull-down experiments were fractionated on a Superdex 200 HR 10/30 column (GE Healthcare Life Sciences) as described below. Every second fractions (18-38) were concentrated by TCA precipitation, dissolved in $30 \mu \mathrm{L}$ of SDS sample buffer, run on a $9 \%$ SDS-PAGE gel, and analyzed by immunoblotting.

Strep-tagged transgenic proteins and their derivatives were affinity purified on a Strep-Tactin spin column (IBA) in the presence of complete protease inhibitor cocktail (Roche) according to the manufacturer's recommendation. Bound proteins were eluted with $2 \mathrm{mM}$ D-biotin ( $\mathrm{pH} 8.0$ ), run on a 9\% SDS-PAGE gel, and immunoblotted.

Separation of the $26 \mathrm{~S}$ proteasome and the extraproteasomal proteins was performed in proteasome buffer supplemented with complete protease inhibitor cocktail on a Superdex 200 HR 10/30 column. Protein extracts prepared from 60 transgenic third-instar larvae were loaded onto the column and subsequently fractionated $(0.1 \mathrm{~mL} / \mathrm{min})$ into $0.35 \mathrm{~mL}$ aliquots. A $35 \mu \mathrm{L}$ aliquot from every second fraction $(18-40)$ was run on a $9 \%$ SDS-PAGE gel and analyzed by immunoblotting.

Superdex 200 fraction (32) prepared from Flag-p54-overexpressing third-instar larvae (Flag-p54/ $\Delta$ p54-da-Gal4) was loaded into anti-FlagM $\mathrm{M}_{2}$ agarose beads (Sigma-Aldrich) preequilibrated with proteasome buffer in the presence of complete protease inhibitor cocktail (Roche). After being incubated for $30 \mathrm{~min}$, beads were washed extensively, and bound proteins were eluted with $200 \mu \mathrm{g} / \mathrm{mL}$ Flag peptide, concentrated, run on an $8 \%$ SDS-PAGE gel, and analyzed by immunoblotting.

DNA Constructs. To create GST-tagged recombinant Rad23-NTH and Dsk2-NTH, the following cloning procedure was used (Figure S2 of the Supporting Information). The cDNA encoding Drosophila Rad23 (Rad23-PA, CG1836) or Dsk2 (Ubqn-PA, CG14224) was subcloned from pFlag-Mac/Rad23 or pFlag-Mac/Dsk2 recombinant plasmids ${ }^{10}$ to the pGEX-4T.1 vector (GE Healthcare) in frame with the $\mathrm{N}$-terminal GST tag. To remove the C-terminal half of $\operatorname{Rad} 23$ (377-1245 bp) or Dsk2 (758-1644 bp), pGEX-4T.1/Rad23 was digested with Eco72IBglII, blunted with Klenow polymerase, and self-circularized, while pGEX-4T.1/Dsk2 was digested with SalI and XhoI and selfcircularized. Final constructions (designated pGEX-4T.1/Rad23NTH and pGEX-4T.1/Dsk2-NTH) were used for bacterial expression of GST-Rad23-NTH or GST-Dsk2-NTH proteins carrying the UBL of Rad23 or Dsk2, respectively.

Transgenic Constructs. Construction of Flag-p54 (p54 cDNA, $1-1188 \mathrm{bp}$ ) was described previously. ${ }^{14}$ To make Flag$\mathrm{p} 54-\Delta \mathrm{K}$, the $1-1143 \mathrm{bp}$ segment of Flag-p54 was amplified via polymerase chain reaction (PCR) with GoTaq DNA polymerase (Promega, Mannheim, Germany) $\left[25\right.$ times $\left(95^{\circ} \mathrm{C}\right.$ for $30 \mathrm{~s}$, $60{ }^{\circ} \mathrm{C}$ for $30 \mathrm{~s}$, and $72{ }^{\circ} \mathrm{C}$ for $\left.30 \mathrm{~s}\right)$ ] using the primer pair 5'-AAGGTACCTGCAGATCCCATGGTTCT-3' (Fw1) and 5'-ATGCTGTCGGTTCACTCAACTAACTCGAGG-3' (Rev1). The PCR product was digested with Acc65I-XhoI and ligated into the pFlag-Mac vector (Sigma Aldrich) in frame with the N-terminal Flag tag.

Construction of Strep-CTH (p54 cDNA, 614-1188 bp) was described previously. ${ }^{10}$ To make Strep-CTH- $\Delta \mathrm{K}$, the $614-$ 1143 bp segment was amplified via PCR from the Strep-CTH template using the primer pair 5'-GTTCGAATTCGGTGTAGATCCCAACGAGGA-3' (Fw2) and 5'-ATGCTGTCGGTTCACTCAACTAACTCGAGG-3' (Rev2). The PCR product was digested with Bsp119I-XhoI and ligated into the pASKIBA5 vector (IBA) in frame with the N-terminal Strep tag. All constructs were verified by DNA sequencing.

Finally, Flag-p54, Flag-p54- $\Delta \mathrm{K}$, Strep-CTH, and Strep-CTH$\Delta \mathrm{K}$ cDNA cassettes were cloned into the $\mathrm{pP}[\mathrm{UAST}]$ shuttling vector. The plasmid constructs were injected into $\mathrm{w}^{1118}$ embryos, and transformed flies were selected according to standard procedures. ${ }^{15}$

All the constructs were verified by DNA sequencing. Plasmid sequences are available upon request.

Fly Work. Fly stocks were cultured at $25{ }^{\circ} \mathrm{C}$ on standard Drosophila food. All genetic and morphological markers used were described previously. ${ }^{16,17}$ The UAS-Gal4 system $^{18}$ was 


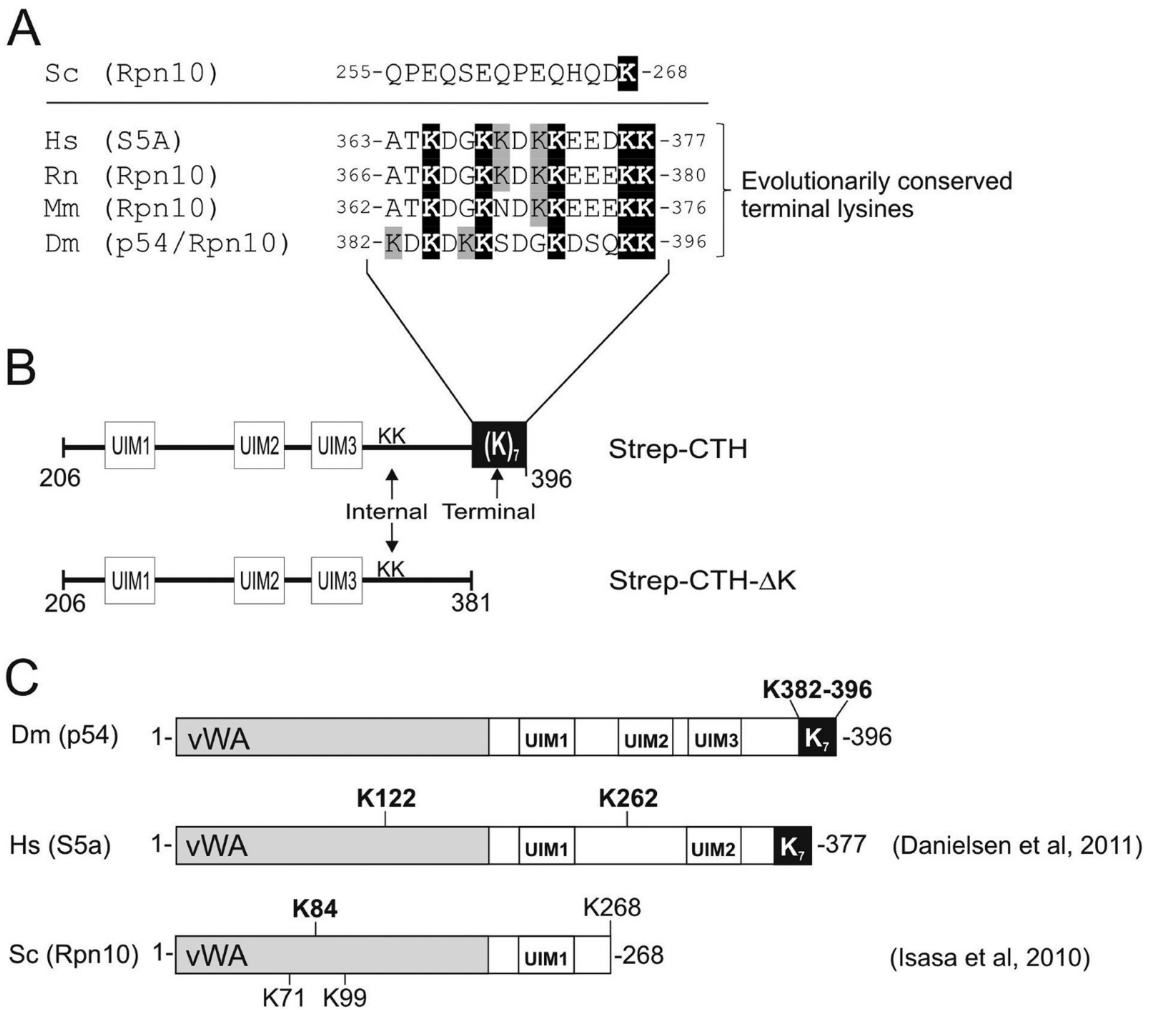

Figure 1. Drosophila p54 and its orthologues. (A) Sequence alignment of the C-terminal lysine cluster of p54 and its higher eukaryotic [human (Hs), mouse $(\mathrm{Mm})$, and rat $(\mathrm{Rn})]$ orthologues. As a comparison, the C-terminus of the yeast $(\mathrm{Sc}) \mathrm{Rpn} 10$ is shown. (B) Schematic view of Strep-CTH and its deletion derivative $($ Strep-CTH- $\Delta \mathrm{K})$. The positions of the two internal lysines $(\mathrm{KK})$ and the evolutionarily conserved terminal lysines $\left(\mathrm{K}_{7}\right)$ are marked by black arrows. (C) Domain structure of p54 and its human (S5a) and yeast (Rpn10) orthologues. Lysines found to be ubiquitylated in yeast Rpn10 and human S5a are highlighted.

applied to induce the overexpression of the appropriate transgenes by crossing transgenic lines to the da-Gal4 driver stock. ${ }^{14}$ Lethal phase analysis was performed as described previously. ${ }^{19}$

\section{Transgenic Lines}

\begin{tabular}{|c|c|}
\hline da-Gal4 & $w ;$ If $/ \mathrm{CyO}$; da-Gal4 \\
\hline Flag-p54/ $\Delta$ p54-da-Gal4 & $w ; P($ UAST-Flag-p54); $\Delta p 54$, da-Gal4 \\
\hline Flag-p54- $\Delta \mathrm{K} / \Delta \mathrm{p} 54-$ da-Gal4 & $w ; P(U A S T-F l a g-p 54-\Delta K) ; \Delta p 54$, da-Gal4 \\
\hline Strep-CTH & $w ;$ If/CyO; $P($ UAST-Strep-CTH $)$ \\
\hline Strep-CTH/da-Gal4 & $w ;$ If/CyO; $P($ UAST-Strep-CTH $) / d a-G a l 4$ \\
\hline Strep-CTH- $\Delta \mathrm{K}$ & $w ;$ If/CyO; $P(U A S T-S t r e p-C T H-\Delta K)$ \\
\hline Strep-CTH- $\Delta \mathrm{K} / \mathrm{da}-\mathrm{Gal} 4$ & $w$; If/CyO; P(UAST-Strep-CTH- $\Delta K) / d a-G a l 4$ \\
\hline
\end{tabular}

Image Processing. X-ray film and Coomassie Brilliant Blue-stained gels were scanned, and images were converted to grayscale without any manipulation. Final images were compiled using Corel Draw X4.

\section{RESULTS}

Identification of the Ubiquitylation Sites of p54-CTH. Our previous work revealed the multiubiquitylation (or multiple monoubiquitylation) of the $\mathrm{CTH}$ in a Drosophila transgenic line overexpressing the Step-tagged version of the $\mathrm{CTH} .{ }^{10}$ As ubiquitylation occurs on lysine residues, we analyzed the sequence of the $\mathrm{CTH}$ and found two internal (K329 and $\mathrm{K} 332)$ and seven terminal (K382, K384, K386, K387, K391, K395, and K396) lysines (Figure 1A). Multiple alignments of the protein sequences of different p54 orthologues indicated that, while the internal lysines do not show conservation (Figure S1 of the Supporting
Information), the terminal lysines (five of seven) are evolutionarily conserved in higher eukaryotes (Figure $1 \mathrm{~A}$ and Figure S1 of the Supporting Information) from Drosophila to humans. We therefore established a new transgenic Drosophila line overexpressing the Strep-tagged version of a CTH derivative from which the cluster of terminal lysines was deleted [Strep-CTH- $\Delta \mathrm{K}$ (6141143 bp segment of p54 cDNA)]. The expression of the transgenic protein was induced by a standard procedure, using the UAS-Gal4 system. ${ }^{18}$ Strep-tagged proteins were affinity-purified on a Strep-Tactin column. Figure 2 presents the Strep-Tactin chromatography patterns of total larval protein extracts of three different transgenic lines: the noninduced full-length Strep-CTH (panel A), the da-Gal4-induced full-length CTH (Strep-CTH/ da-Gal4, panel B), and the da-Gal4-induced Strep-CTH- $\Delta$ K (Strep$\mathrm{CTH}-\Delta \mathrm{K} / \mathrm{da}-\mathrm{Gal} 4$, panels $\mathrm{C}$ and $\mathrm{D})$. The total larval protein extract (I-input), proteins that flow through the affinity column (FT), and the affinity-purified proteins (B-bound) were fractionated on a $9 \%$ SDS-PAGE gel and immunoblotted with an anti-p54 monoclonal antibody, which recognizes only the CTH of p54 (Figure 2A-C), or with a polyclonal antibody that recognizes only the NTH of p54 (Figure 2D).

All the ubiquitylated derivatives of Strep-CTH previously characterized by mass spectrometry ${ }^{10}$ are induced by the da-Gal 4 driver (compare panels A and B of Figure 2). For the da-Gal4induced Strep-CTH- $\Delta \mathrm{K}$ transgenic animals, apart from the expressed transgenic protein, only a faint band of the nonspecifically bound full-length endogenous p54 eluted from the affinity column (marked by a white asterisk in panels A-D); the ubiquitylated derivatives of the full-length $\mathrm{CTH}$ are missing. The faint band in panel $\mathrm{C}$ is definitely not a modified form of Strep-CTH- $\Delta \mathrm{K}$ 

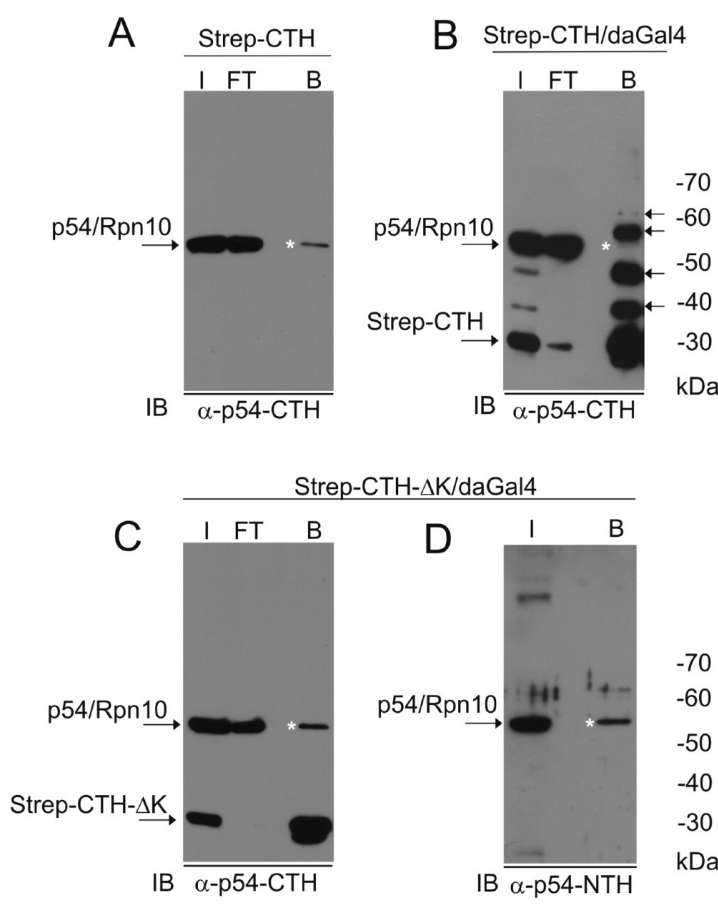

Figure 2. Strep-CTH is ubiquitylated on the conserved C-terminal lysine cluster. (A) The third-instar larval protein extract of the noninduced Strep-CTH transgenic line was loaded onto a Strep-Tactin affinity column. An aliquot of the input total extract (I), the flowthrough fraction (FT), and the fraction bound to the affinity matrix and eluted with D-biotin (B) were fractionated on a 9\% SDS-PAGE gel and analyzed by immunoblotting with a monoclonal anti-p54 antibody recognizing the $\mathrm{CTH}$ of p54. The asterisk in all four panels marks the nonspecifically bound endogenous p54 protein. (B) Affinity purification of Strep-CTH and its derivatives from the third-instar larval protein extract of Strep-CTH/da-Gal4 transgenic animals. Details are described for panel A. Ubiquitylated CTH derivatives identified previously by mass spectrometry ${ }^{10}$ are marked by black arrows at the right. (C) Affinity purification of Strep-CTH- $\Delta \mathrm{K}$ from the third-instar larval protein extract of Strep-CTH- $\Delta \mathrm{K} / \mathrm{da}-\mathrm{Gal} 4$ transgenic animals. Details are described for panel A. (D) The band marked with an asterisk in all four panels is the nonspecifically bound endogenous p54 and not a modified form of the $\mathrm{CTH}$, because it is recognized by a polyclonal antibody specific for the N-terminal half of p54 (anti-p54-NTH).

because it reacted with a polyclonal antibody specific for the NTH of p54 (panel D). The transgenic Strep-CTH- $\Delta \mathrm{K}$ protein appeared as a closely spaced doublet. The difference in the molecular mass of the p54 immunoreactive doublet band is slight, indicating that, not ubiquitylation, but either a specific proteolytic cleavage or some other unidentified postsynthetic modification is responsible for the appearance of the doublet (Figure 2C). With a lighter exposure (data not shown), it is clear that the Strep-CTH depicted in panel $\mathrm{B}$ also appears as a doublet. The lack of the ubiquitylated CTH bands in the Strep-CTH- $\Delta \mathrm{K}$ protein indicates that the site of ubiquitylation within the p54-CTH protein is not the two internal lysines but the evolutionarily conserved terminal lysine cluster.

Ubiquitylation of the Terminal Lysines of CTH Abolishes Its Interaction with the UBA-UBL-Containing Extraproteasomal Polyubiquitin Receptors. The UBAUBL-containing extraproteasomal polyubiquitin receptors play an essential role in the targeting of the polyubiquitylated proteins to the $26 \mathrm{~S}$ proteasomes. ${ }^{20}$ The UBA (ubiquitin-associated) domain is responsible for the selective recognition and binding of the polyubiquitin chain, while the UBL (ubiquitin-like) domain interacts with the UIM motifs of $\mathrm{p} 54^{14}$ or its orthologues; this interaction ensures the targeting of the polyubiquitylated proteins to the $26 \mathrm{~S}$ proteasome. ${ }^{21}$ The presence of both the ubiquitylated and nonubiquitylated forms of CTH in our transgenic Drosophila lines allowed the analysis of the interaction of these forms with the UBL-domain present in the NTH of the Dsk2 and Rad23 proteins, the two major extraproteasomal polyubiquitin receptors functioning in D. melanogaster. ${ }^{10}$ We used the NTH of Dsk2 and Rad23 to avoid the intra- and intermolecular interactions between the UBA and UBLs and to avoid the cross-interactions mediated by the UBA/polyubiquitylated protein/UIMs. In pull-down experiments, GST (which served as a control), GST-Dsk2-NTH, or GSTRad23-NTH was immobilized on Glutathione Sepharose. Strep$\mathrm{CTH}$ and its ubiquitylated derivatives, prepurified on a StrepTactin column, were loaded onto the GST-, GST-Dsk2-NTH-, or GST-Rad23-NTH-charged beads. Proteins that eluted with reduced glutathione were analyzed by immunoblotting with an anti-p54 antibody. As shown in Figure 3, the nonubiquitylated

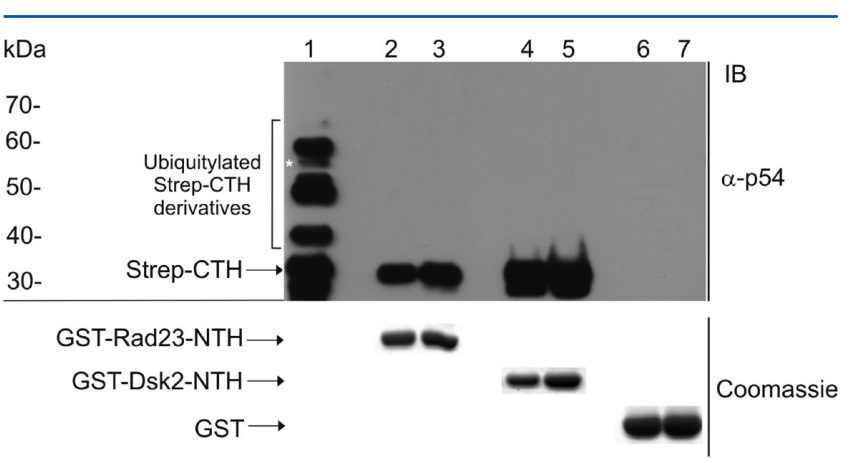

Figure 3. Ubiquitylation interrupts the interaction of $\mathrm{CTH}$ with the UBL of Dsk2 and Rad23. Affinity-purified Strep-CTH and its derivatives (lane 1) were loaded onto a GST-Rad23-NTH-, GSTDsk2-NTH-, or GST-charged Glutathione Sepharose column; $20 \%$ (lane 2) and $30 \%$ (lane 3) of proteins that eluted from the GSTRad23-NTH column, 20\% (lane 4) and 30\% (lane 5) of proteins that eluted from the GST-Dsk2-NTH column, or $20 \%$ (lane 6) and $30 \%$ (lane 7) of proteins that eluted from the GST column were fractionated on a 9\% SDS-PAGE gel and analyzed by immunoblotting with a monoclonal anti-p54 antibody. The quantity of GST-Rad23NTH, GST-Dsk2-NTH, or GST proteins in eluted fractions is demonstrated by Coomassie Brilliant Blue staining. Nonspecifically bound endogenous p54 is marked with an asterisk in lane 1 .

CTH bound efficiently and specifically to both the Dsk2-NTH and the Rad23-NTH affinity columns, but not even traces of the ubiquitylated $\mathrm{CTH}$ derivatives were recovered from these columns. Besides the $\mathrm{CTH}$ and its ubiquitylated derivatives, there are no other proteins in stoichiometric amounts in the affinity-purified $\mathrm{CTH}$ fraction ${ }^{10}$ that could interact only with the ubiquitylated derivatives of the $\mathrm{CTH}$ to block their specific interaction with the extraproteasomal polyubiquitin receptors. This observation indicates that ubiquitylation of $\mathrm{CTH}$ drastically reduces or abolishes its affinity for the UBL of the extraproteasomal polyubiquitin receptors, channeling these modified protein derivatives toward a nonproteasomal cellular route.

Full-Length p54 Is Also Ubiquitylated. The extraproteasomal localization of the CTH may mimic the presumed extraproteasomal state of subunit p54, but obviously, there is no direct indication that it is a realistic model for the intracellular fate of the full-length proteasomal subunit. In the purified Drosophila $26 \mathrm{~S}$ proteasome, we have never detected the ubiquitylated form of 


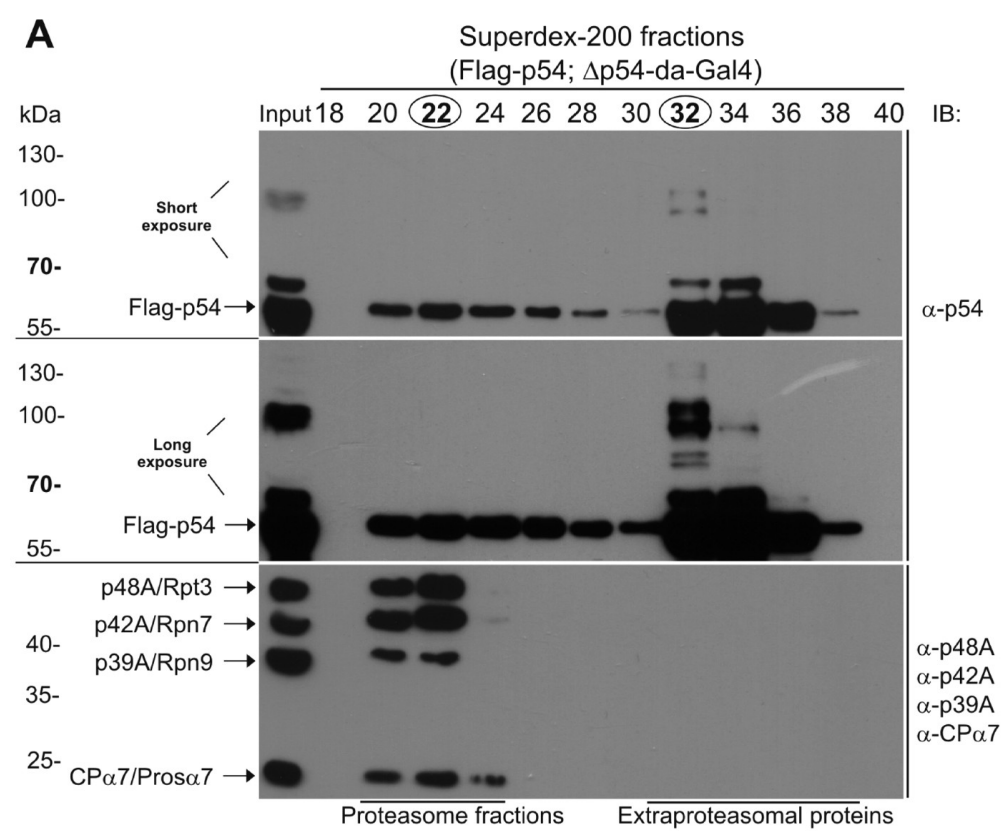

B

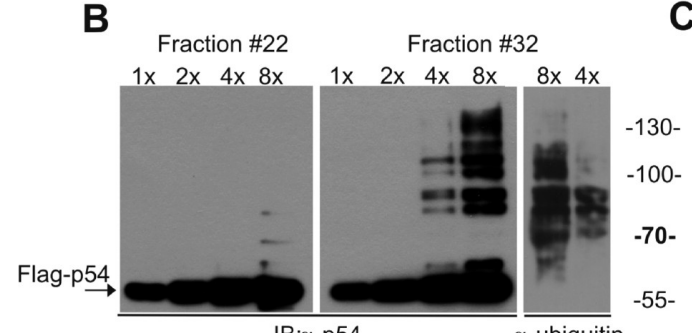

C
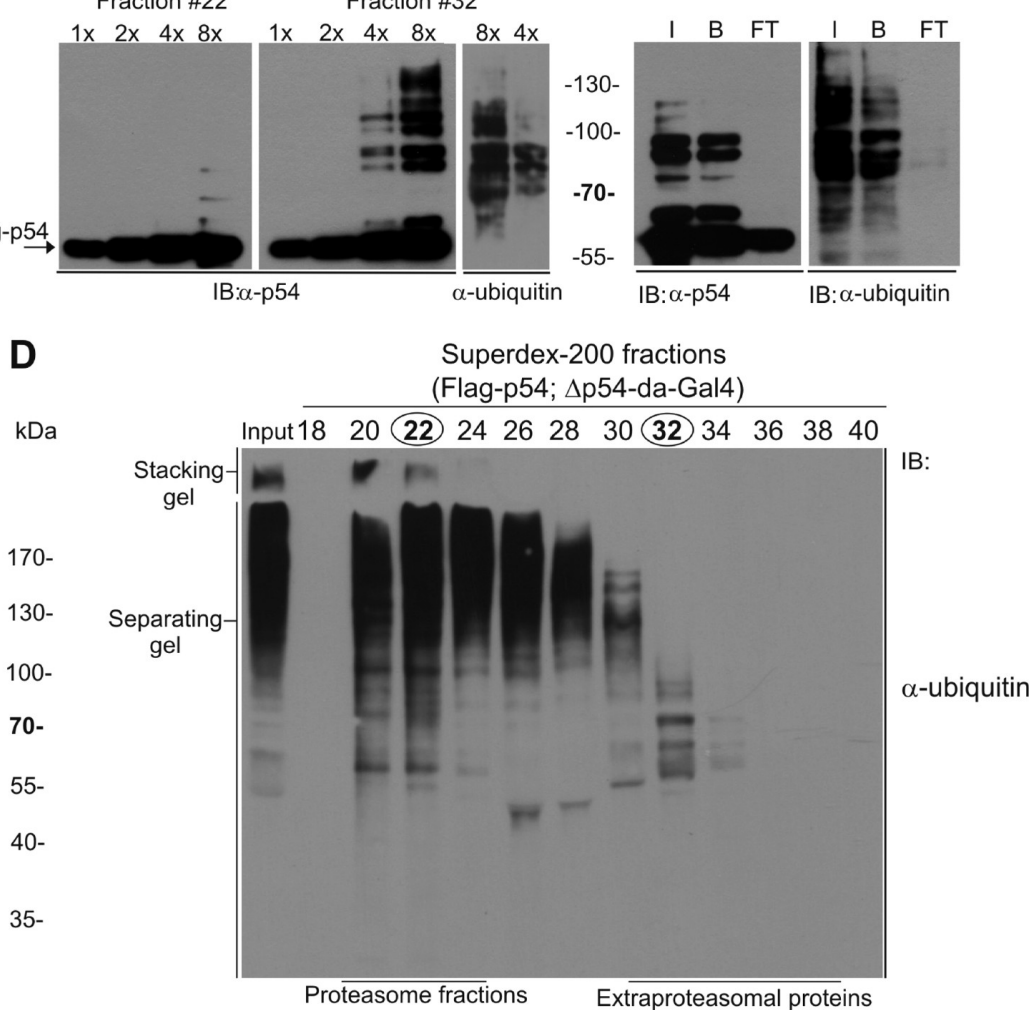

Figure 4. Distribution of the ubiquitylated Flag-p54 derivatives. (A) The third-instar larval protein extract of the Flag-p54/ $\Delta$ p54-da-Gal4 transgenic line was fractionated on a Superdex 200 sizing column. The total extract (Input) and every second of the Superdex 200 fractions (18-40) were separated on a 9\% SDS-PAGE gel and analyzed by immunoblotting with a monoclonal anti-p54 antibody. A short exposure and a long exposure of the immunoblot are presented. The same fractions were analyzed with a mixture of monoclonal antibodies specific for the RP and CP subunits (bottom). (B) Increasing quantities of fractions 22 (the peak fraction of the $26 \mathrm{~S}$ proteasome) and 32 (the peak fraction of the extraproteasomal ubiquitylated Flag-p54 derivatives) were fractionated on an 8\% SDS-PAGE gel and analyzed by immunoblotting with monoclonal anti-p54 or polyclonal anti-ubiquitin antibodies. (C) The ubiquitin chains detected in panel B are physically linked to Flag-p54. Proteins of fraction 32 were affinity purified on Flag beads, and an aliquot of the input fraction (I), the fraction eluted from the Flag beads (B), and an aliquot of the flow-through (FT) were analyzed by immunoblotting with anti-p54 and anti-ubiquitin antibodies. (D) Distribution of polyubiquitylated proteins in the Superdex 200 fractions analyzed on a 7\% SDS-PAGE gel by immunoblotting with anti-ubiquitin antibodies. To detect the highly polyubiquitylated proteins, the stacking gel was blotted, too. 
subunit p54, and in a freshly prepared Drosophila total protein extract (from embryo, larva, pupa, or adult), only trace amounts of the extraproteasomal p54 protein can be found. ${ }^{10}$ Detection of ubiquitylation of full-length p54 has therefore failed to date in wildtype animals. To boost the chance of detecting ubiquitylation of fulllength p54, a transgenic Drosophila line in which the expression of the Flag-tagged version of full-length p54 can be induced by the UAS-Gal4 system was generated. We have previously shown that overexpression of the Flag-p54 transgene can fully complement the lethal phenotype of the Drosophila p54 null mutant, from which the single copy gene encoding the p54 protein has been deleted. ${ }^{19}$ This is direct proof that the Flag-p54 protein is fully functional. ${ }^{14}$ Flagp54 was overexpressed in a modified p54 null mutant genetic background (Flag-p54/ $\Delta$ p54-da-Gal4 ${ }^{14}$ ), and therefore, all the p54 proteins that are incorporated into the $26 \mathrm{~S}$ proteasome or appeared extraproteasomally are transgenic Flag-p54. For separate analysis of the proteasome-bound Flag-p54 protein and the extraproteasomal pool of Flag-p54 protein, a total protein extract prepared from thirdinstar larvae of the Flag-p54/ $\Delta$ p54-da-Gal4 transgenic line was fractionated by size on a Superdex 200 sizing column, and the chromatography fractions were analyzed by immunoblotting with either the anti-p54 antibody (Figure 4A) or a mixture of monoclonal antibodies recognizing the RP (p48A/Rpt3, p42A/ Rpn7, and p39A/Rpn9) and CP (CP $\alpha 7 / \operatorname{Pros} \alpha 7)$ subunits of the $26 \mathrm{~S}$ proteasome. In fractions $20-24$, where the $26 \mathrm{~S}$ proteasome is eluted (Figure 4A), the p54 subunit appears as a single band and only a trace amount of the higher-molecular mass derivative of p54 was visible in overloaded sample (Figure 4B, fraction 22). This is in sharp contrast with the immunoblotting pattern of extraproteasomal p54 in fractions of monomeric proteins (Figure 4A, fractions 32-36). Above the extraproteasomal full-length Flag-p54 protein, one very strong and a series of weaker p54 immunoreactive bands appeared. Similar results were obtained with the anti-Flag antibody (data not shown). The strong band was $\sim 8 \mathrm{kDa}$ larger than fulllength Flag-p54. When fraction 32 was analyzed with anti-ubiquitin, all the characteristic bands larger than Flag-p54 were immunoreacted (Figure 4B). The higher the molecular mass of the Flag-p54 derivatives, the stronger their relative reactivity with the antiubiquitin antibody, indicating that the increase in molecular mass is due to a higher level of ubiquitylation. To prove that the detected ubiquitin chains are physically linked to the Flag-p54 protein, fraction 32 was affinity purified on Flag beads, and the proteins bound by the Flag beads or the proteins that passed through the affinity column were analyzed by immunoblotting with anti-p 54 and anti-ubiquitin antibodies. As shown in Figure 4C, the anti-p54 reactive and the anti-ubiquitin reactive proteins were efficiently bound by the Flag beads and the immunoreactive proteins were depleted in the flow-through fraction. Our data indicate that the extraproteasomal Flag-p54 protein is extensively multiubiquitylated, while only very modest modification occurs on the proteasomebound form.

The distribution of the polyubiquitylated proteins in the Superdex 200 chromatography fractions was analyzed by immunoblotting with the anti-ubiquitin antibody. The majority of the polyubiquitylated proteins coeluted with the $26 \mathrm{~S}$ proteasomes (Figure 4D), and a significant amount eluted as very high molecular mass protein.

Ubiquitylation of Flag-p54 Abolishes Its Interaction with the UBA-UBL-Containing Extraproteasomal Polyubiquitin Receptors. The functional impact of Flag-p54 ubiquitylation on its interaction with the UBL of Dsk2 and Rad23 was analyzed in pull-down assays. In these experiments, GST-Dsk2-NTH or GST-Rad23-NTH was immobilized on
Glutathione Sepharose beads and a total protein extract prepared from Flag-p54/ $\Delta$ p54-da-Gal4 third-instar larvae was loaded onto the affinity columns. Proteins that passed through the affinity columns and those that were eluted by reduced glutathione were size fractionated on a Superdex 200 column (Figure 5A,B). The Dsk2-NTH-bound fraction contained the majority of those Flag-p54 protein molecules that were assembled into the $26 \mathrm{~S}$ proteasome, and only a small amount of extraproteasomal monomer Flag-p54 (Figure 5A) was present. Just the opposite was true for the proteins that appeared in the flow-through fraction of the Dsk2-NTH affinity column (Figure 5A). In this fraction, only very few proteasome-bound proteins and a large amount of extraproteasomal Flag-p54 and its ubiquitylated forms were present. These experiments indicate that the affinity of Dsk2-NTH is much higher for the $26 \mathrm{~S}$ proteasome-assembled Flag-p54 than for the monomer Flag-p54.

To test the affinity of Dsk2-NTH for the ubiquitylated or nonubiquitylated extraproteasomal Flag-p54 proteins, fraction 32 from Superdex 200 chromatography was analyzed. This fraction is completely free of $26 \mathrm{~S}$ proteasomes (Figure $4 \mathrm{~A}$ ). To make the analysis fully comparable to the experiment demonstrating the interaction of GST-Dsk2-NTH with the affinity-purified Strep-CTH and its ubiquitylated derivatives (Figure 3), Flag-p54 and its derivatives were first affinity purified from fraction 32 on anti-FlagM $\mathrm{M}_{2}$ beads, and the GSTDsk2-NTH pull-down experiment was performed using these highly purified protein fractions. As shown in Figure 6A while a substantial amount of nonubiquitylated monomer Flag-p54 bound to GST-Dsk2-NTH, all its ubiquitylated derivatives passed through the column. Thus, the affinity of the UBL of Dsk2 is highest for RP-assembled Flag-p54 and lower for extraproteasomal Flag-p54, and it does not interact with the ubiquitylated forms of Flag-p54. This observation confirms the results obtained with the Strep-CTH protein; ubiquitylation drastically reduces the affinity of Dsk2-NTH for full-length p54 or its $\mathrm{CTH}$.

The results obtained with the protein fractions that bound to or passed through the Rad23-NTH affinity column were slightly different. Rad23-NTH has a much lower affinity for proteasome-assembled and extraproteasomal Flag-p54 (Figure 5B). In the absence of competing 26S proteasomes, Rad23-NTH, like the Dsk2-NTH, bound nonubiquitylated Flag-p54 but could not interact with its ubiquitylated derivatives (Figure 6B).

Our original idea to use the NTH (UBL) of Dsk2 for the purification of p54-containing Drosophila proteasomes was also applied by other research groups using our DNA constructs and protocols. Sakata and colleagues have recently published their results; ${ }^{22}$ they clearly demonstrate the power and advantages of this technique as an efficient tool for one-step purification of structurally and functionally intact $26 \mathrm{~S}$ proteasomes suitable for electron cryomicroscopy studies and answering fundamental questions in this field.

The Conserved Terminal Lysines Are Ubiquitylated in Full-Length p54. The role of the terminal lysines of fulllength p54 was analyzed in a transgenic animal overexpressing a Flag-tagged derivative of full-length p54 from which the cluster of terminal lysines had been deleted (Flag-p54- $\Delta \mathrm{K}$ ). When Flag-p $54-\Delta \mathrm{K}$ was expressed in a modified $\Delta \mathrm{p} 54$ genetic background (Flag-p54- $\Delta \mathrm{K} / \Delta \mathrm{p} 54-\mathrm{da}-\mathrm{Gal} 4$ ), a large fraction of the expressed Flag-p $54-\Delta \mathrm{K}$ protein was incorporated into the $26 \mathrm{~S}$ proteasome, demonstrating that the lack of the terminal lysines does not impair its assembly with the RP. Neither the 
A

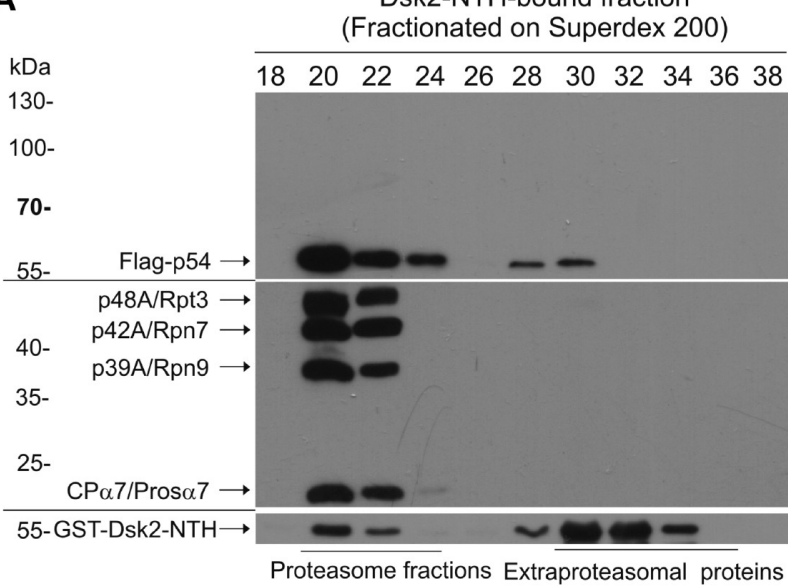

Proteasome fractions Extraproteasomal proteins

Rad23-NTH-bound fraction

B

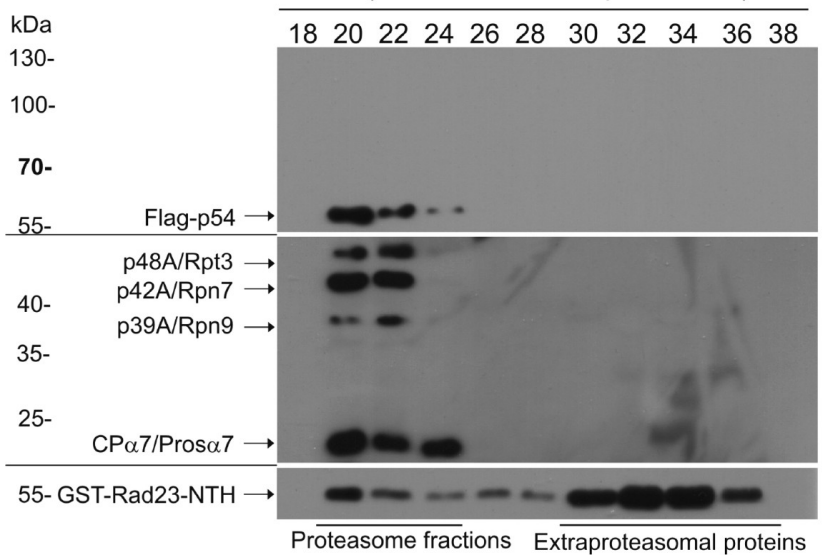

Dsk2-NTH flow-through

(Fractionated on Superdex 200)

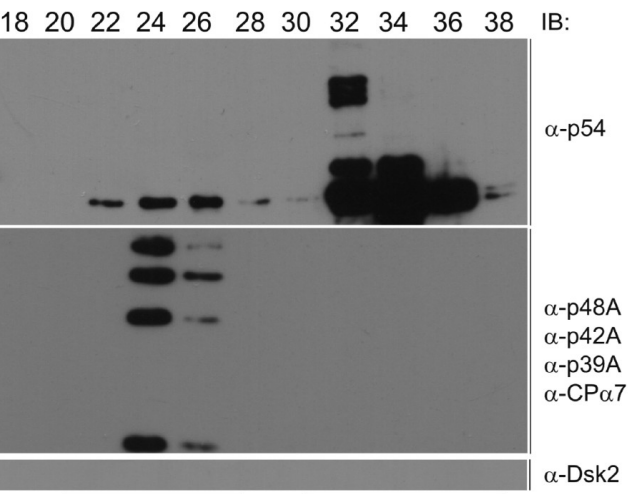

Proteasome fractions Extraproteasomal proteins
Rad23-NTH flow-through (Fractionated on Superdex 200)

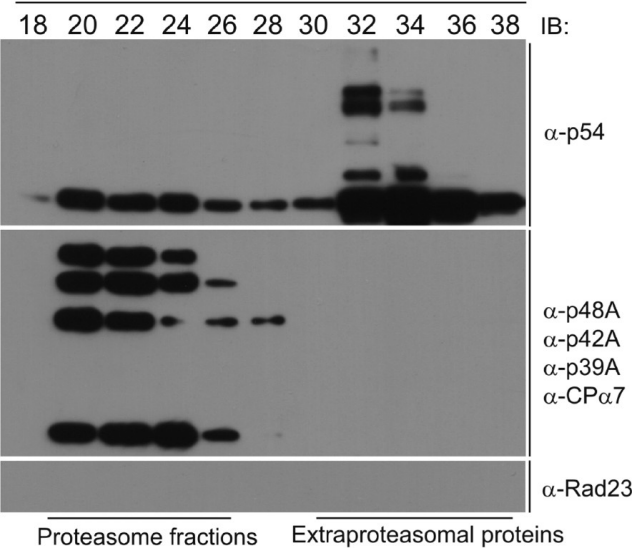

Figure 5. Interaction of Dsk2-NTH and Rad23-NTH with proteasome-assembled and extraproteasomal Flag-p54. (A) Protein fractions that bound or passed through the Dsk2-NTH affinity column were size fractionated on a Superdex 200 column. Chromatography fractions were separated on a 9\% SDS-PAGE gel and analyzed by immunoblotting with an anti-p54 antibody (top), with a mixture of proteasome specific antibodies (middle) or the anti-Dsk2 antibody (bottom). (B) Same analysis as described in panel A with the fractions of the Rad23-NTH affinity purification. The same fractions were analyzed with a mixture of proteasome specific antibodies or with the anti-Rad23 antibody.

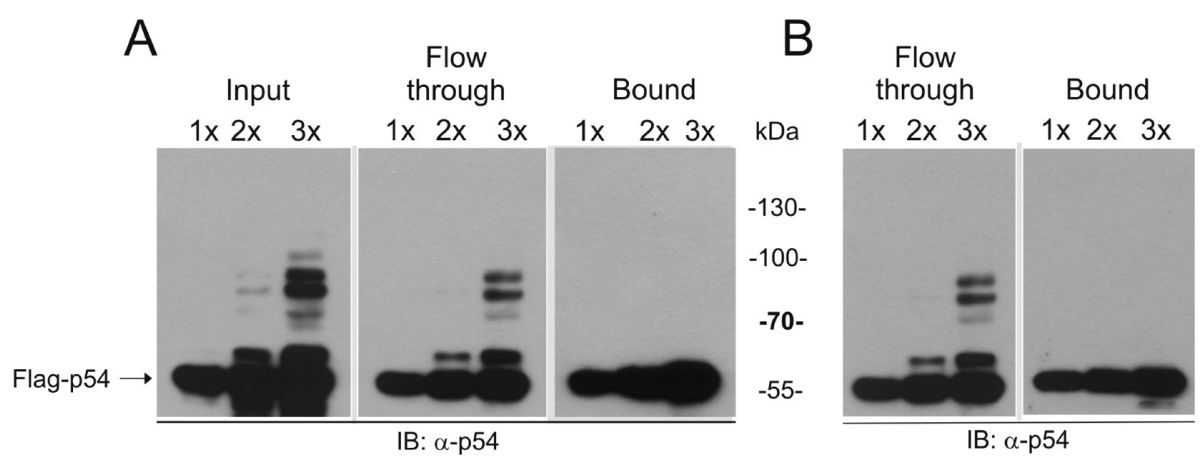

Figure 6. Interaction of Dsk2-NTH and Rad23-NTH with ubiquitylated and nonubiquitylated extraproteasomal Flag-p54. (A) Proteins of Superdex fraction 32 were affinity purified on Flag beads, and the purified proteins were loaded onto a GST-Dsk2-NTH column. Increasing amounts of the Flag bead-purified proteins (Input), proteins that flow through the Dsk2-NTH column (Flow through), and proteins that interact with Dsk2-NTH (Bound) were fractionated on an $8 \%$ SDS-PAGE gel and immunoblotted with the anti-p54 antibody. (B) Proteins of Superdex fraction 32 were affinity purified on Flag beads, and the purified proteins (shown in panel A) were loaded onto a GST-Rad23-NTH column. Increasing amounts of proteins that flow through the Rad23-NTH column (Flow through) and proteins that interact with Rad23-NTH (Bound) were fractionated on an $8 \%$ SDS-PAGE gel and immunoblotted with the anti-p54 antibody.

$\mathrm{RP}$-assembled nor the extraproteasomal Flag-p $54-\Delta \mathrm{K}$ protein was ubiquitylated (Figure 7), indicating that, as in Strep-CTH, the evolutionarily conserved terminal lysines are ubiquitylated within full-length p54, and the removal of these terminal lysines does not enhance the ubiquitylation of other internal lysine residues. 


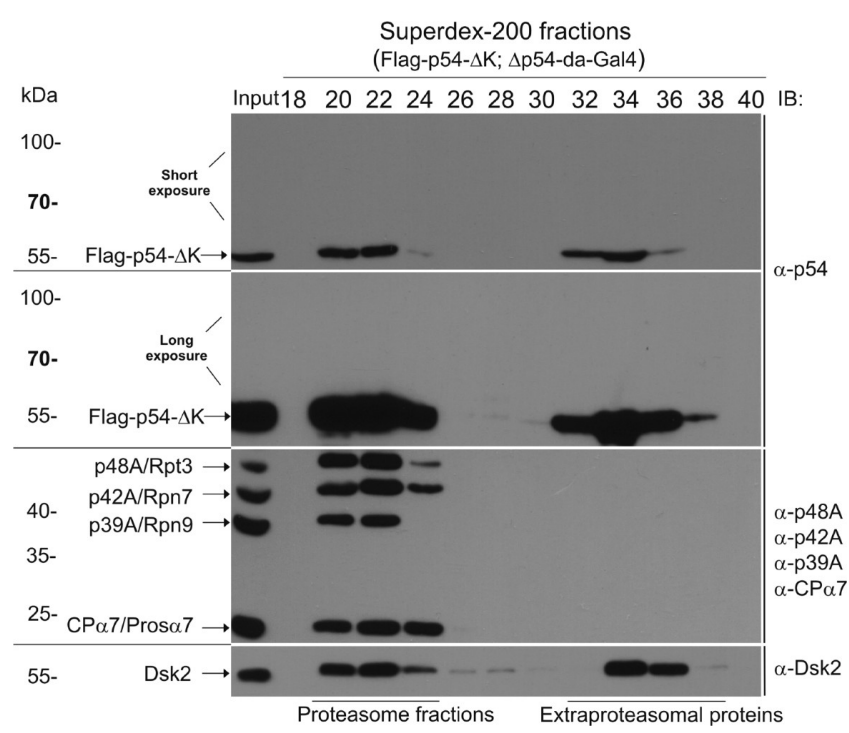

Figure 7. Flag-p54- $\Delta \mathrm{K}$ protein is not ubiquitylated. The third-instar larval protein extract of Flag-p54- $\Delta \mathrm{K} / \Delta \mathrm{p} 54-\mathrm{da}-\mathrm{Gal} 4$ transgenic animals was fractionated on a Superdex 200 sizing column. An aliquot of the total extract (Input) and every second Superdex 200 fractions (18-40) were separated on a 9\% SDS-PAGE gel and analyzed by immunoblotting with the monoclonal anti-p54 antibody. A short exposure and a long exposure of the immunoblot are presented. The same fractions were analyzed with a mixture of proteasome specific antibodies or with the anti-Dsk2 antibody.

$\Delta$ p54 proteasomes are deficient in Dsk2 binding, and the expression of the transgenic Flag-p54 protein restores the interaction of Dsk2 with the proteasomes. ${ }^{14}$ When expressed on a $\Delta$ p54-da-Gal4 genetic background, the Flag-p54- $\Delta \mathrm{K}$ protein, like full-length Flag-p54, can fully restore Dsk2 binding (Figure 7), indicating that the lack of the terminal lysine cluster does not impair the function of the UIM motifs. Expression of the Flag-p $54-\Delta \mathrm{K}$ transgenic protein in the $\Delta \mathrm{p} 54$-da-Gal4 mutant genetic background resulted in a shift in the lethal phase of the $\Delta$ p54 mutation. The $\Delta$ p54 mutation causes polyphasic larvalpupal lethality, ${ }^{19}$ but these mutant animals die as early pupae and

Table 1. Mutant Mortality during Development

\begin{tabular}{lcc} 
& \multicolumn{2}{c}{ mortality $(\%)$} \\
\cline { 2 - 3 } developmental stage & $\Delta \mathrm{p} 54-\mathrm{da}-\mathrm{Gal} 4$ & Flag-p54- $\Delta \mathrm{K} / \Delta \mathrm{p} 54-\mathrm{da}-\mathrm{Gal} 4$ \\
embryo & 0 & 0 \\
L1 larva & 40 & 0 \\
L2 larva & 20 & 0 \\
L3 larva & 13 & 0 \\
pupa & 27 & 9 \\
pharate adult & 0 & 91 \\
adult & 0 & 0
\end{tabular}

never reach the pharate adult stage. Flag-p54- $\Delta \mathrm{K} / \Delta \mathrm{p} 54-\mathrm{da}-\mathrm{Gal} 4$ animals survived until the late pharate adult stage (Table 1 ). The failure to rescue the lethality of the $\Delta \mathrm{p} 54$ mutation may indicate that the ubiquitylated form of the extraproteasomal p54 protein has an essential function during the developmental transition from pupa to adult.

\section{DISCUSSION}

Our in vivo studies indicate that, in its extraproteasomal state, the p54 subunit of the $26 \mathrm{~S}$ proteasome is multiubiquitylated.
We could detect only very modest ubiquitylation in the RPassembled p54. The site of ubiquitylation both in the CTH of p54 and in the full-length subunit is the cluster of evolutionarily conserved terminal lysines, because deletion of the cluster abolishes the in vivo ubiquitylation of p54. These terminal lysines should be sterically accessible inside the RP to the appropriate ubiquitin ligase enzyme(s), because subunit p54 is responsible for the interactions with polyubiquitylated proteins and polyubiquitin receptors, which require substantial mobility and flexibility for this segment of the subunit. The highly limited ubiquitylation of subunit p54 inside the $26 \mathrm{~S}$ proteasome may suggest that this modification initiates the dissociation of subunit p54. This assumption may provide a regulatory clue about how the dissociation of subunit p54 is controlled.

One of the main biological functions of subunit p54, i.e., the interaction of its UIM motifs with the UBL of the extraproteasomal polyubiquitin receptors, is abolished following the ubiquitylation; this modification may channel the subunit toward a nonproteasomal cellular route. The use of different tags in our previous work (Strep-tag ${ }^{10}$ ) and this work (Flag tag) excludes the possibility that tag-dependent nonspecific ubiquitylation was observed. The specific in vivo postsynthetic modification of p54 in its extraproteasomal state strongly suggests that this subunit of the RP dissociates in vivo and participates as a shuttling factor in a hitherto unidentified cellular function.

In vivo multiubiquitylation of Rpn10, the yeast orthologue of Drosophila p54, was recently described and characterized. ${ }^{2,24}$ Isasa and colleagues demonstrated that a similar pattern of multiple monoubiquitylation can be achieved in vitro. In this in vitro experiment, lysines $\mathrm{K} 71, \mathrm{~K} 84$, and $\mathrm{K} 268$ were identified as the sites of monoubiquitylation. K71 and the major ubiquitylated site K84 are located within the N-terminal vWA domain of Rpn10. This domain mediates the binding of this subunit to the $\mathrm{RP}^{12}$. $\mathrm{K} 268$ is at the very C-terminus of the protein. In vivo, K84 was found to be the predominant monoubiquitylated form. Interestingly, multiubiquitylation occurred both on the proteasomeassembled and on the extraproteasomal Rpn10 protein. The physiological relevance of the ubiquitylation was supported by the observation that the serious growth defect of the $\operatorname{rpn} 10 \Delta \operatorname{rad} 23 \Delta$ yeast double mutation could not be rescued by a plasmid-encoded Rpn10 in which K71, K84, K99, and K268 were mutated; however, wild-type Rpn10 completely rescued this defect. An in vitro assay demonstrated that multiubiquitylated Rpn10 exhibits a low affinity for polyubiquitin conjugates.

In a recently published high-throughput work, ubiquitylation of lysine K122 (in the vWA domain) and K262 (between UIM1 and UIM2) of S5a, the human orthologue of Rpn10/ p54, was revealed by random mass spectrometric analysis. ${ }^{25}$ These lysines, however, do not show conservation in yeast or Drosophila (Figure S1 of the Supporting Information). Although ubiquitylation of endogenous p54 has been indicated in Drosophila neuronal cells by ${ }^{\mathrm{Bio}} \mathrm{Ub}$ conjugate specific pulldown experiments, ${ }^{26}$ the site and biological relevance of the modification have not been elucidated.

The molecular mechanisms of the ubiquitylation of the yeast and Drosophila proteasomal polyubiquitin receptor subunits differ significantly. In yeast, $\mathrm{K} 84$ is the main in vivo monoubiquitylated lysine residue, ${ }^{24}$ which is located in the vWA domain. This lysine residue is modified in the $\mathrm{RP}$-assembled and extraproteasomal Rpn10 proteins. In Drosophila, there is only very modest monoubiquitylation of the RP-assembled p54; this is in sharp contrast with the extensive multiple monoubiquitylations of the extraproteasomal p54. Deletion of the evolutionarily conserved terminal 
lysines of p54 completely suspends the ubiquitylation, indicating that the internal lysine residues are not ubiquitylated, not even following removal of the potentially competing terminal lysines. The structural features of ubiquitylation of the proteasomal ubiquitin receptor subunits of the yeast and Drosophila 26S proteasome are basically different, suggesting that the functional consequences of this postsynthetic modification will also be diverse. The most significant functional consequence of Flag-p54 ubiquitylation in Drosophila is the inability of the ubiquitylated subunit to interact with the UBL of the extraproteasomal polyubiquitin receptors. This may be due to an intramolecular snap-back and binding of the terminally located ubiquitin moieties to the UIM motifs of p54, resulting in their self-inhibition. The interaction of the ubiquitylated forms of Rpn10 with the UBL of polyubiquitin receptors has not been studied in yeast. ${ }^{23,24}$

The significantly different details of the multiubiquitylation processes of the Rpn10 and p54 subunits extend the hitherto known divergences in the mechanism with regard to how these orthologues participate in the targeting of polyubiquitylated proteins to the $26 \mathrm{~S}$ proteasome. The most conspicuous difference in their function is that deletion of Rpn10 or Dsk2 in the yeast is not lethal and causes only modest changes in intracellular proteolysis, ${ }^{11,27}$ whereas the deletion of Drosophila p54 ${ }^{19}$ and mouse Rpn $10^{28}$ or the downregulation of Drosophila Dsk $2^{14}$ is absolutely lethal. The second main difference is that in yeast UBLcontaining Dsk2 binds to the Rpn1 subunit of the $\mathrm{RP}^{29}$ and not to Rpn10, and Dsk2 can interact only with $\Delta \mathrm{Rpn} 10$ proteasomes, not with the wild-type one. ${ }^{30}$ In Drosophila, just the opposite is true: Dsk2 does not interact with $\Delta \mathrm{p} 54$ proteasomes, but the interaction can be fully restored by complementing the $\Delta \mathrm{p} 54$ deletion with the Flag-p54 transgenic protein. ${ }^{14}$ The difference in the C-terminal amino acid sequence of Rpn10 as compared with those of higher eukaryotic orthologues may explain the divergence in their modes of action. The amino acid sequence of Rpn10 is highly homologous to those of its higher eukaryotic orthologues, except that a fairly long C-terminal sequence present in all higher eukaryotic orthologues (128 amino acids in the case of Drosophila) is missing from yeast Rpn10 (Figure S1 of the Supporting Information). The cluster of conserved lysines that serves an important function in Drosophila, as described in our study, is missing from Rpn10. The C-terminal lysine present in Rpn10 is far from the conserved terminal sequences of the higher eukaryotic orthologues. Considering the high level of sequence conservation in the N-terminal and internal segments of Rpn10 and its orthologues, just how this sequence difference evolved remains for the time being a matter of speculation. The C-terminal sequence present only in higher eukaryotes may possibly have been appended to an early subunit configuration later during the evolution, ensuring a new function of the subunit.

\section{ASSOCIATED CONTENT}

\section{S Supporting Information}

Amino acid sequence alignment of human S5a (Hs), Drosophila p54 (Dm), and yeast Rpn10 (Sc) proteins (Figure S1) and cloning scheme of Rad23-NTH and Dsk2-NTH (Figure S2). This material is available free of charge via the Internet at http://pubs.acs.org.

\section{AUTHOR INFORMATION}

\section{Corresponding Author}

*E-mail: udvardy@brc.hu. Telephone: +36-62-599-664. Fax: +36-62-433-506.

\section{Funding}

This work was supported by the Institute of Biochemistry (Biological Research Centre of the Hungarian Academy of Sciences).

\section{Notes}

The authors declare no competing financial interest.

\section{ACKNOWLEDGMENTS}

We are thankful to Margit Pál providing help in fly work. We used FlyBase and PubMed as the sources of information and literature.

\section{ABBREVIATIONS}

NTH, N-terminal half; CTH, C-terminal half; UBA, ubiquitinassociated domain; UBL, ubiquitin-like domain; UIM, ubiquitin-interacting motif; vWA, von Willebrand factor type A domain; GST, glutathione $S$-transferase.

\section{REFERENCES}

(1) Ravid, T., and Hochstrasser, M. (2008) Diversity of degradation signals in the ubiquitin-proteasome system. Nat. Rev. Mol. Cell Biol. 9, 679-690.

(2) Braun, B. C., Glickman, M., Kraft, R., Dahlmann, B., Kloetzel, P. M., Finley, D., and Schmidt, M. (1999) The base of the proteasome regulatory particle exhibits chaperone-like activity. Nat. Cell Biol. 1, 221-226.

(3) Strickland, E., Hakala, K., Thomas, P. J., and DeMartino, G. N. (2000) Recognition of misfolding proteins by PA700, the regulatory subcomplex of the $26 \mathrm{~S}$ proteasome. J. Biol. Chem. 275, 5565-5572.

(4) Verma, R., Aravind, L., Oania, R., McDonald, W. H., Yates, J. R., Koonin, E. V., and Deshaies, R. J. (2002) Role of Rpn11 metalloprotease in deubiquitination and degradation by the $26 \mathrm{~S}$ proteasome. Science 298, 611-615.

(5) Yao, T. T., and Cohen, R. E. (2002) A cryptic protease couples deubiquitination and degradation by the proteasome. Nature 419, 403-407.

(6) Kohler, A., Cascio, P., Leggett, D. S., Woo, K. M., Goldberg, A. L., and Finley, D. (2001) The axial channel of the proteasome core particle is gated by the Rpt2 ATPase and controls both substrate entry and product release. Mol. Cell 7, 1143-1152.

(7) Hochstrasser, M. (2006) Lingering mysteries of ubiquitin-chain assembly. Cell 124, 27-34.

(8) Collins, G. A., and Tansey, W. P. (2006) The proteasome: A utility tool for transcription? Curr. Opin. Genet. Dev. 16, 197-202.

(9) Haracska, L., and Udvardy, A. (1995) Cloning and sequencing a non-ATPase subunit of the regulatory complex of the Drosophila 26-S protease. Eur. J. Biochem. 231, 720-725.

(10) Lipinszki, Z., Kiss, P., Pal, M., Deak, P., Szabo, A., HunyadiGulyas, E., Klement, E., Medzihradszky, K. F., and Udvardy, A. (2009) Developmental-stage-specific regulation of the polyubiquitin receptors in Drosophila melanogaster. J. Cell Sci. 122, 3083-3092.

(11) vanNocker, S., Sadis, S., Rubin, D. M., Glickman, M., Fu, H. Y., Coux, O., Wefes, I., Finley, D., and Vierstra, R. D. (1996) The multiubiquitin-chain-binding protein Mcb1 is a component of the 265 proteasome in Saccharomyces cerevisiae and plays a nonessential, substrate-specific role in protein turnover. Mol. Cell. Biol. 16, 60206028.

(12) vanNocker, S., Deveraux, Q., Rechsteiner, M., and Vierstra, R. D. (1996) Arabidopsis MBP1 gene encodes a conserved ubiquitin recognition component of the $26 \mathrm{~S}$ proteasome. Proc. Natl. Acad. Sci. U.S.A. 93, 856-860.

(13) Kiss, P., Szabo, A., Hunyadi-Gulyas, E., Medzihradszky, K. F., Lipinszki, Z., Pal, M., and Udvardy, A. (2005) $\mathrm{Zn}^{2+}$-induced reversible dissociation of subunit Rpn10/p54 of the Drosophila 26 S proteasome. Biochem. J. 391, 301-310.

(14) Lipinszki, Z., Pál, M., Nagy, O., Deák, P., Hunyadi-Gulyas, E., and Udvardy, A. (2011) Overexpression of Dsk2/dUbqln results in 
severe developmental defects and lethality in Drosophila melanogaster that can be rescued by overexpression of the p54/Rpn10/S5a proteasomal subunit. FEBS J. 278, 4833-4844.

(15) Spradling, A. C. (1986) in Drosophila: A Practical Approach (Roberts, D., Ed.) IRL Press, Oxford, U.K.

(16) Ashburner, M. (1989) Drosophila: A Laboratory Handbook, Cold Spring Harbor Laboratory Press, Plainview, NY.

(17) Lindsley, D. L., and Zimm, G. G. (1992) The Genome of Drosophila melanogaster, Academic Press, San Diego.

(18) Duffy, J. B. (2002) GAL4 system in Drosophila: A fly geneticist's Swiss army knife. Genesis 34, 1-15.

(19) Szlanka, T., Haracska, L., Kiss, I., Deak, P., Kurucz, E., Ando, I., Viragh, E., and Udvardy, A. (2003) Deletion of proteasomal subunit S5a/Rpn10/p54 causes lethality, multiple mitotic defects and overexpression of proteasomal genes in Drosophila melanogaster. J. Cell Sci. 116, 1023-1033.

(20) Hartmann-Petersen, R, and Gordon, C. (2004) Ubiquitinproteasome system: Proteins interacting with the $26 \mathrm{~S}$ proteasome. Cell. Mol. Life Sci. 61, 1589-1595.

(21) Hurley, J. H., Lee, S., and Prag, G. (2006) Ubiquitin-binding domains. Biochem. J. 399, 361-372.

(22) Sakata, E., Bohn, S., Mihalache, O., Kiss, P., Beck, F., Nagy, I., Nickell, S., Tanaka, K., Saeki, Y., Förster, F., and Baumeister, W. (2012) Localization of the proteasomal ubiquitin receptors Rpn10 and Rpn13 by electron cryomicroscopy. Proc. Natl. Acad. Sci. U.S.A., doi: $10.1073 /$ pnas.1119394109.

(23) Crosas, B., Hanna, J., Kirkpatrick, D. S., Zhang, D. P., Tone, Y., Hathaway, N. A., Buecker, C., Leggett, D. S., Schmidt, M., King, R. W., Gygi, S. P., and Finley, D. (2006) Ubiquitin chains are remodeled at the proteasome by opposing ubiquitin ligase and deubiquitinating activities. Cell 127, 1401-1413.

(24) Isasa, M., Katz, E. J., Kim, W., Yugo, V., Gonzalez, S., Kirkpatrick, D. S., Thomson, T. M., Finley, D., Gygi, S. P., and Crosas, B. (2010) Monoubiquitination of RPN10 Regulates Substrate Recruitment to the Proteasome. Mol. Cell 38, 733-745.

(25) Danielsen, J. M. R., Sylvestersen, K. B., Bekker-Jensen, S., Szklarczyk, D., Poulsen, J. W., Horn, H., Jensen, L. J., Mailand, N., and Nielsen, M. L. (2011) Mass Spectrometric Analysis of Lysine Ubiquitylation Reveals Promiscuity at Site Level. Mol. Cell. Proteomics 10, M110.003590.

(26) Franco, M., Seyfried, N. T., Brand, A. H., Peng, J., and Mayor, U. (2011) A novel strategy to isolate ubiquitin conjugates reveals wide role of ubiquitination during neural development. Mol. Cell. Proteomics 10, M110.002188.

(27) Funakoshi, M., Sasaki, T., Nishimoto, T., and Kobayashi, H. (2002) Budding yeast Dsk2p is a polyubiquitin-binding protein that can interact with the proteasome. Proc. Natl. Acad. Sci. U.S.A. 99, 745750.

(28) Hamazaki, J., Sasaki, K., Kawahara, H., Hisanaga, S., Tanaka, K., and Murata, S. (2007) Rpn10-mediated degradation of ubiquitinated proteins is essential for mouse development. Mol. Cell. Biol. 27, 66296638.

(29) Elsasser, S., Gali, R. R., Schwickart, M., Larsen, C. N., Leggett, D. S., Muller, B., Feng, M. T., Tubing, F., Dittmar, G. A. G., and Finley, D. (2002) Proteasome subunit Rpn1 binds ubiquitin-like protein domains. Nat. Cell Biol. 4, 725-730.

(30) Matiuhin, Y., Kirkpatrick, D. S., Ziv, I., Kim, W., Dakshinamurthy, A., Kleifeld, O., Gygi, S. P., Reis, N., and Glickman, M. H. (2008) Extraproteasomal Rpn10 Restricts Access of the Polyubiquitin-Binding Protein Dsk2 to Proteasome. Mol. Cell $32,415-425$. 